\title{
Efecto protector de los extractos acuoso e hidroetanólico de hojas de Bixa orellana (achiote) sobre los indicadores no enzimáticos de hepatotoxicidad en ratas
}

Oscar Huamán, Miguel Sandoval, Elsa Béjar, Víctor Tarazona

Centro de Investigación de Bioquímica y Nutrición, Facultad de Medicina, UNMSM

Objetivos: Determinar el efecto de extractos acuoso e hidroetanólico de hojas de Bixa orellana (achiote) sobre los indicadores no enzimáticos de hepatotoxicidad, en ratas sometidas a paracetamol.

Diseño: Experimental.

Institución: Centro de Investigación de Bioquímica y Nutrición, Facultad de Medicina, UNMSM.

Material biológico: 35 ratas machos de 3 meses de edad.

Intervenciones: Las ratas fueron distribuidas aleatoriamente en 5 grupos, que recibieron vía peroral, por 10d: controles positivo y negativo ( $\mathrm{NaCl} 0,9 \mathrm{~g} / \mathrm{dL}$ ), silimarina $300 \mathrm{mg} / \mathrm{kg}$, extracto acuoso (500 mg/kg) y extracto hidroetanólico $50 \%$ $(500 \mathrm{mg} / \mathrm{kg}) ;$ al $5^{\circ}$ día se administró paracetamol (400 mg/kg) peroral, excepto el control negativo, previo a un ayuno de $24 \mathrm{~h}$. Bajo anestesia con éter se realizó la punción cardiaca para extraer sangre.

Principales medidas de resultados: Ratio hepático (peso hígado/peso animal x 100), bilirrubina total, directa e indirecta $(\mathrm{mg} / \mathrm{dL})$, especies reactivas al ácido tiobarbitúrico $(\mathrm{nmol} / \mathrm{mL})$. Comparaciones al control positivo $(\mathrm{p}<0,05)$.

Resultados: Los extractos disminuyeron el ratio hepático. Los grupos silimarina y extractos disminuyeron significativamente la bilirrubina total e indirecta. La bilirrubina directa solo disminuyó significativamente con el extracto hidroetanólico. Los TBARS disminuyeron significativamente en suero e hígado en los grupos con extracto acuoso e hidroetanólico, respectivamente.

Conclusiones: Los extractos acuoso e hidroetanólico de hojas de Bixa orellana (achiote) presentaron efecto hepatoprotector frente al paracetamol a dosis tóxica, en ratas.

Palabras clave: Bixa orellana, TBARS, bilirrubina, indice hepático, hepatoprotector.

\section{Efecto del zumo de fruto Opuntis ficus indica (tuna), variedad morada (ZFT), sobre los niveles de lipoperoxidación, en ratas sometidas a intoxicación con paracetamol}

\author{
Carlos Sánchez, Oscar Huamán, Sotomayor Guillermo, Zoraida Huamán
}

Departamento Académico de Nutrición, UNMSM

Objetivos: Determinar el efecto del zumo de pulpa del fruto Opuntia ficus indica (tuna), variedad morada, sobre los niveles de especies reactivas al ácido tiobarbitúrico, en ratas sometidas a intoxicación con paracetamol.

Diseño: Experimental.

Institución: Departamento Académico de Nutrición, UNMSM.

Material biológico: 36 ratas albinas Holtzman machos.

Intervenciones: Las ratas fueron mantenidas con dieta balanceada y agua ad libitum. Se las distribuyó aleatoriamente en seis grupos, recibiendo el siguiente tratamiento por diez días: control negativo y positivo (suero fisiológico), silimarina (300 mg/kg de pc), y zumo entero (ZE), zumo al medio (ZM), zumo al cuarto (ZC). Al quinto día recibió paracetamol (400 $\mathrm{mg} / \mathrm{kg} \mathrm{pc}$ ) por cinco días.

Principales medidas de resultados: Especie reactiva al ácido tiobarbiturico ( $\mathrm{nmol} / \mathrm{mL})$, porcentaje de índice hepático. Resultados: Los niveles de TBARS en tejido hepático y suero disminuyeron en los grupos que recibieron ZE y ZM, en comparación al grupo control positivo, de forma significativa $(p>0,05)$.

Conclusión: Los zumos entero y al medio de pulpa del fruto Opuntia ficus indica (tuna) presentaron efecto protector frente a la lipoperoxidación inducida por intoxicación con paracetamol.

Palabras clave: Opuntia ficus indica, lipoperoxidación, paracetamol. 